\title{
Disjunctive discourse referents in French Sign Language*
}

\author{
Jeremy Kuhn \\ Institut Jean Nicod, CNRS, ENS, \\ EHESS, PSL University
}

\begin{abstract}
Disjoined noun phrases, like indefinites, may introduce indeterminate discourse referents. Disjunction provides more flexibility in some respects than indefinites, though, as the two disjuncts may bear different morphological features, and a disjunctive discourse referent may have a split antecedent. Sign language, too, has been shown to bear on arguments pertaining to discourse anaphora. Notably, discourse referents may be established at locations in the signing space (loci), closely paralleling the use of variables in dynamic semantics. Here, we compare several theories of disjunctive anaphora and of space in sign language with new data from French Sign Language (LSF). We argue that loci must be mediated by a featural layer that iconically preserves mereological properties.
\end{abstract}

Keywords: dynamic semantics, disjunction, sign language

\section{Introduction}

It is well known that indefinites can introduce discourse referents (Kamp 1981; Heim 1982). In (1), the indefinite someone introduces a discourse referent that is retrieved by the pronoun them. This discourse referent is indeterminate-whichever person asks the question, that is the person that I thank later.

(1) When someone asks a good question, I make sure to thank them later.

But, indeterminate discourse referents are not specific to only indefinite quantifiers. Notably, disjunction is another construction that may generate an indeterminate discourse referent. In (2), the pronoun it varies over donkeys and horses, depending on what Mary sees in a given situation.

(2) When Mary sees a donkey or a horse, she waves to it.

* The research presented in this paper benefitted from discussion with Scott AnderBois, Simon Charlow, Emmanuel Chemla, Emil Enguehard, Michael Erlewine, Kathryn Davidson, Carlo Geraci, Polly Jacobson, Tory Sampson, Philippe Schlenker, and Ronnie Wilbur. The research leading to these results received support from ERC H2020 grant 788077 (Orisem), and ANR-17-EURE-0017 (FrontCog). 
To some extent, these facts should not be surprising. After all, disjunction is equivalent to indefinite quantification over a domain of two individuals.

On the other hand, disjunction provides more flexibility than indefinites in several respects. First, the two disjuncts may bear different morphosyntactic features. This may introduce grammatical conflicts that affect later pronominal reference. In (3), one disjunct is masculine and the other is feminine. As a result, neither a masculine nor a feminine pronoun is grammatical; one needs to use a pronoun not specified for gender such as them.

(3) Whenever Mary sees a boy or a girl, she waves to $\{*$ him/*her/them $\}$.

Second, a disjunctive referent may have a split antecedent, arising from disjoined sentences. In (4), unlike in (2), there is no DP node that can be identified as the antecedent of the pronoun $i t$, but the pronoun nevertheless can retrieve a disjunctive discourse referent.

(4) Whenever Mary catches a fish or John traps a rabbit, Bill cooks it.

As we will see in Section 2, these kinds of data have fed into existing debates in the literature regarding the best way to represent anaphoric dependencies.

In sign languages, anaphoric dependencies have been shown to involve the use of space. Specifically, in most sign languages, a DP may be established at a locusa point in the horizontal signing space in front of the signer. In later discourse, referential expressions such as pronouns and anaphora may be directed back to this locus in order to retrieve the relevant individual, as illustrated in Figure 1. Notably, this use of loci seems to intuitively parallel the use of variables in theories of dynamic semantics, motivating some researchers to posit that loci are in fact an overt morphosyntactic spell-out of logical variables (Lillo-Martin \& Klima 1990; Schlenker 2011).

In this paper, I show that the simplest dynamic analysis of loci faces challenges on many examples with disjunction. Such examples oblige us to refine both theories of disjunctive anaphora and of space in sign language. In light of these challenges, I propose a revised system on which (a) spatial loci are mediated by a featural layer; (b) in spoken and sign language, pronouns may denote sums of discourse referents;

(c) the spatial features of these sum individuals are iconically determined.

Methodology The data in this paper come from French Sign Language (LSF: Langue des Signes Française), based on consulting sessions with one Deaf native signer of LSF. Following the playback method of Schlenker (2011), all tested sentences were video recorded; the resulting videos were later played back to the consultant for grammaticality and semantic judgments on several separate occasions. 

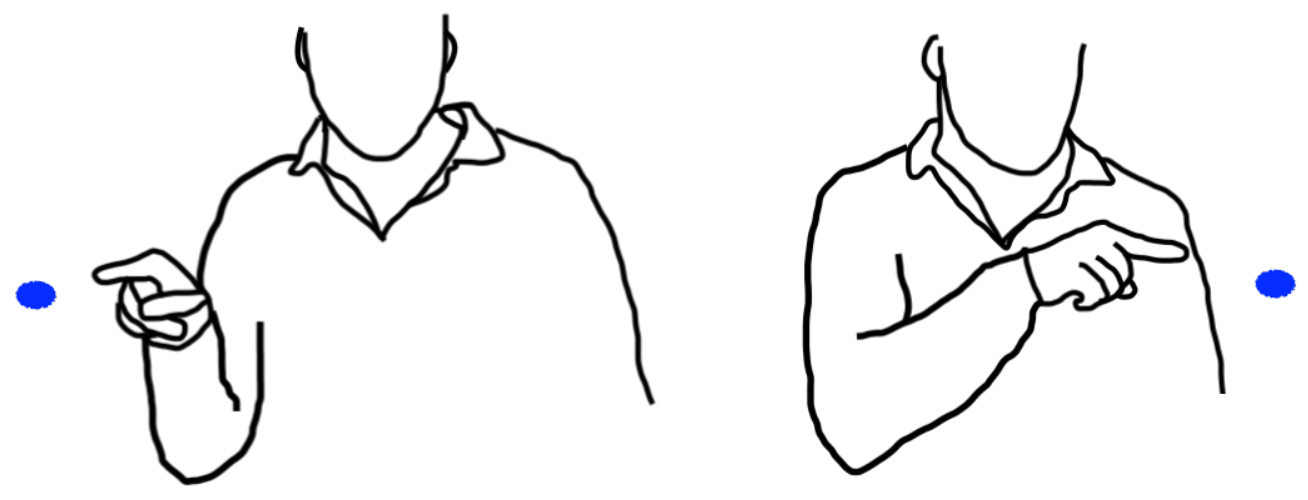

Figure 1 Directing a pronoun (IX) to a locus on the right or the left of the signer

Grammaticality judgments consisted of ratings on a seven point scale (7=best). Semantic judgments consisted of answers (in LSF) to open questions about the meaning of the sentence; these explanations were also video recorded. Full numerical ratings for the sentences presented in this paper are provided in the Appendix.

Following standard conventions, we gloss LSF signs using their closest English translation in small capitals. Pronouns (signed with an extended index finger) are glossed IX. Loci are glossed with lowercase letters; IX-a is thus a pronoun at locus a.

\section{Background: Dynamic theories}

Historically, there have been two main approaches to analyzing discourse reference: dynamic semantics and E-type theories. On theories of dynamic semantics, DPs introduce discourse referents as indices in a data structure. Pronouns retrieve the values at these indices (Kamp 1981; Heim 1982; Groenendijk \& Stokhof 1991; Dekker 1993). In (5), for example, the first sentence introduces $\mathrm{Al}$ at $y$ and a value at $x$ that varies across assignment functions. The pronouns in the second sentence retrieve the values of $x$ and $y$ in each assignment function.

(5)

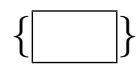

a. $\mathrm{A} \operatorname{girl}^{x}$ waved $\mathrm{Al}^{y}$ over.

$$
\{x \mapsto \text { amy, } y \mapsto \text { al }, x \mapsto \text { ann, } y \mapsto \text { al }, \ldots\}
$$

b. She $x$ gave $\operatorname{him}_{y}$ a flower.

On E-type theories, propositions are identified with minimal situations. Pronouns are elided definite descriptions that identify a unique participant in a given situation (Evans 1980; Heim 1990; Stone 1992; Elbourne 2005). In (6), for example, the first sentence introduces a minimal situation in which a girl waved $\mathrm{Al}$ over, and no other 
information is included. In the second sentence, she retrieves the unique girl in that situation, and he the unique boy.

a. ${ }^{S}$ [A girl waved Al over].

b. She $\langle$ girl in $S\rangle$ gave him $\langle$ boy in $S\rangle$ a flower.

In this context, Stone (1992) argues that sentences involving disjunction may provide evidence in favor of E-type theories. On the one hand, dynamic theories have no problem analyzing a discourse like (7), repeated from (2). Of note, the string a donkey or a horse is itself a DP, so it may introduce a discourse referent which can be recovered by the pronoun it. On the other hand, Stone argues that dynamic theories have a problem with sentences like (8), repeated from (4). The two DPs introduce discourse referents at $x$ and $y$, but there is no single index that can be retrieved by the pronoun to access the disjunctive referent.

(7) When Mary sees $\left[[\text { a donkey }]^{x} \text { or }[\text { a horse }]^{y}\right]^{z}$, she waves to it $_{z}$.

(8) Whenever Mary catches $[\mathrm{a} \text { fish }]^{x}$ or John traps [a rabbit $]^{y}$, Bill cooks it??.

In contrast, for E-type theories, there is a unique participant in each minimal situation quantified over by the antecedent clause. In some of these situations, Mary catches a fish; in others, John traps a rabbit. In either case, there is a unique animal that can be retrieved by the pronoun.

Schlenker (2011) provides a defense of dynamic semantics on two fronts. First, based on examples with plural pronouns, he argues that split antecedents are necessary in general to model the anaphoric behavior. In (9), for example, there are at least four different readings of the plural pronoun they, that correspond to different ways of summing the three singular antecedents. Such examples show that a single pronoun can refer to the sum of discourse referents introduced by multiple, syntactically independent antecedents.

(9) $[\text { Each boy }]^{x}$ told [each girl $]^{y}$ that [each teacher $]^{z}$ thought they ${ }_{x+y / x+z / y+z / x+y+z}$ should work together.

Schlenker argues that the same analytic strategy can account for the indeterminate discourse referents introduced by DPs in disjoined sentences. Thus, in (10), the index on the pronoun is $x+y$, denoting the sum of the discourse referents introduced by a fish (if any) with the ones introduced by a rabbit (if any). By the same logic as the E-type theory, in each situation quantified over by whenever, exactly one of these dynamic variables will have a non-empty value, thus licensing the use of the singular pronoun it.

(10) Whenever Mary catches [a fish $]^{x}$ or John traps [a rabbit $]^{y}$, Bill cooks it t $_{x+y}$. 
Second, Schlenker argues that new data from sign language provide evidence in favor of dynamic theories of anaphora. As described in the introduction, sign language loci may disambiguate anaphoric reference. In (11), for example, a pronoun signed towards locus a necessarily denotes the girl, previously established at that index.

\section{(11) GIRL-a BOY-b COMPETE. IX-a WIN. \\ 'A girl and boy competed. The girl won.'}

Of note, Schlenker observes that this pattern holds even when (a) the minimal situation is symmetric, and (b) both NPs apply to both individuals, as in (12). These data pose a challenge for E-type theories, as there is no longer a uniquely described participant in the minimal situation; E-type theories would thus incorrectly predict the pronouns to either be undefined or ambiguous.

EACH-TIME AMERICAN-a AMERICAN-b LIVE TOGETHER, IX-a LIKE IX-b. 'When an American and an American live together, the one likes the other.'

(Schlenker 2011)

Based on such examples, Schlenker (2011) proposes that sign language loci play the role of formal variables in dynamic semantics. On his analysis, a pronoun indexed at a locus denotes the unique discourse referent assigned to that variable by an assignment function, as shown in (13).

$$
\llbracket \mathrm{IX}-\mathrm{a} \rrbracket^{g}=g(\mathrm{a})
$$

In the rest of this paper, I am not going to relitigate the dynamic/E-type debate. Rather, within a dynamic framework, I will look at new, challenging data involving disjunction in French Sign Language (LSF). These data revise our understanding of the relation of loci and dynamic variables.

\section{Challenges to loci as variables}

\subsection{Challenge \#1: Split antecedents}

The main analytical choices so far have been built on two kinds of data. Arguments from disjunction have come primarily from examples with disjoined sentences, as in (10). Arguments from sign language have come primarily from examples involving symmetric situations, as in (12). The interaction of these arguments can be observed by creating examples that combine the two of these properties. The discourse (14), for example, consists of two disjoined sentences, each of which contains a symmetric minimal situation. The discourse is nevertheless unambiguous: the second sentence means that one man or woman asks for the other's phone number. 
Disjunctive discourse referents in LSF

BAR GAY MEANS MAN-a MAN-b MEET GO OR WOMAN-a WOMAN-b MEET GO BAR. IX-a ASK NUMBER TELEPHONE POSS-b.

'At a gay bar, a man and a man meet or a woman and a woman meet. He/she asks his/her number.'

Two important observations can be made about this example. First, we observe that the example exactly mirrors Schlenker's argument against E-type theories. There is no unique man in the minimal situation described by the first sentence and no unique woman in the minimal situation described by the second. The discourse is nevertheless well-defined and unambiguous. This suggests that a dynamic approach is on the right track, even for the kind of disjunctive examples previously used to argue against dynamic semantics.

Second, though, we observe that a single locus indexes multiple variables. Specifically, locus a indexes the discourse referent introduced by MAN-a (call it $x$ ), the discourse referent introduced by WOMAN-a (call it $y$ ), as well as their sum $(x+y)$. This is not possible on the analysis of Schlenker (2011), on which an assignment function maps each locus to a unique individual.

\subsection{Challenge \#2: Disjuncts at different loci}

A second challenge for the above dynamic analysis is related to examples in which the two disjuncts may bear different morphosyntactic features, such as (15), repeated from (3).

(15) Whenever I see a boy or a girl, I wave at $\{*$ him/*her/them $\}$.

Similar examples are also possible in LSF: two disjuncts may be placed at two different loci. In the DP in (16), for example, one disjunct is signed at locus a and the other at locus b. Just like for the English example in (15), such examples may place further restrictions on the form of subsequent pronouns. In such cases, is disjunctive reference possible? And if so, what is the locus of the pronoun?

\section{BOY-a OR GIRL-b}

An initial answer to these questions is provided by Schlenker (2011). Schlenker proposes that directing a pronoun toward either locus generates the inference that, in the local context, an individual indexed at that locus exists. In (17), for example, the two disjuncts are placed at two different loci, a and b. Subsequent pronouns at either locus are slightly degraded, and, to the extent they are possible, they generate a conditional inference: 'The candidate would establish the law if elected.' In the global context, this discourse referent may be empty. But, in the local context generated by modal subordination, the discourse referent is non-empty. 


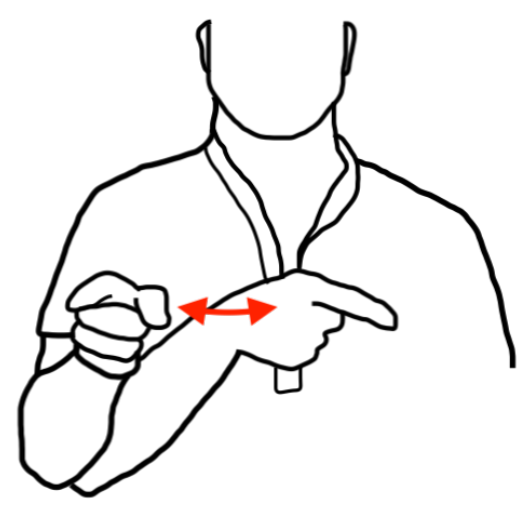

Figure 2 The pronoun IX-alternating

NEXT-YEAR PRESIDENT ELECTION, WHO GOING-TO WIN? PERSON-a BLACK OR PERSON-b ASIAN. THEN IX-b ESTABLISH NEW LAW THEME CITIZEN EQUALITY.

'In next year's presidential election, who is going to win? A Black person or an Asian person. The Asian would then establish a new law on citizen equality.'

By contrast, Schlenker shows that a null pronoun (unspecified for locus) can be used to retrieve the disjunctive referent. In (18), for example, a null pronoun is used in place of an overt pronoun, and a disjunctive discourse referent becomes available: 'Whoever wins will establish the law.' This follows from Schlenker's analysis since null pronouns bear no locus, so are not constrained by the placement of antecedent loci; interpretation of the pronoun thus proceeds as in English.

NEXT-YEAR ELECTION PRESIDENT, WHO WIN? PERSON-a WHITE WIN NOT. PERSON-b BLACK OR PERSON-c ASIAN COMPETE GOING-TO WIN. THEN $\underline{\emptyset}$ LAW ENACT EQUALITY CITIZEN.

'In next year's presidential election, who will win? A white person won't win; a Black person or an Asian person will win. They will then enact a law on citizen equality.'

So far, these examples conform to Schlenker's generalization: that signing a pronoun at a locus generates the inference that an individual at that locus exists in the local context. But a number of other pronominal forms that are not discussed by Schlenker are also possible. First, there is a pronominal form that moves back and forth between the loci indexed by the two disjuncts, as shown in Figure 2. I will gloss this form 'IX-alternating'. 
Example (19) shows that IX-alternating can be used to retrieve a disjunctive referent; the interpretation of the sentence is that whichever of the two wins will establish the law. This differs critically from the minimally different sentence with the dual pronoun THE-TWO, which also moves in space between the two loci, but with a different handshape. As shown in (20), the use of dual pronoun entails the existence of two individuals: a Black candidate and an Asian candidate; regardless of who wins, the two will work together on the new law.

NEXT-YEAR ELECTION PRESIDENT, WHO GOING-TO WIN? IX-a PERSON-a BLACK OR PERSON-b ASIAN. THEN IX-alternating-a,b ESTABLISH NEW LAW EQUALITY CITIZEN.

'In next year's presidential election, who is going to win? A Black person or an Asian person. They will then establish a new law on citizen equality.'

NEXT-YEAR ELECTION PRESIDENT, WHO WIN? BLACK-a OR ASIAN-b. THEN THE-TWO-a,b ESTABLISH LAW EQUALITY CITIZEN.

'In next year's presidential election, who is going to win? A Black person or an Asian person. The two will then establish a law on citizen equality.'

The interpretation of the sentence with the dual pronoun THE-TWO is cleanly accounted for by Schlenker's proposal: the pronoun activates two loci, and there is an inference that two discourse referents exist. But the interpretation of the sentence with IX-alternating is ostensibly a counterexample to the generalization: a pronoun activates two distinct loci without generating the inference that two individuals exist. On the other hand, such examples may still be compatible with Schlenker's generalization if the lexical semantics of the pronoun IX-alternating is itself disjunctive, similar to the English expression he or she, as in (21). Under such an analysis, the pronoun introduces its own disjunctive local context under which each of the two loci is interpreted.

Whenever I see a boy or a girl, he or she waves at me.

Finally, it is possible to retrieve a disjunctive discourse referent by directing a pronoun to the middle of two loci, schematized in Figure 3. In (22), for example, three loci are used to establish three discourse referents: one at locus a, to the right of the signer; a second at locus b, slightly to the left of center; and a third at locus c, far to the left of center. Directing a pronoun generally to the left, to the midpoint of $b$ and $c$, allows a disjunctive discourse referent to be retrieved: whichever of those two people wins, that person will then establish the law. Similar examples can be constructed with only two loci, with a similar disjunctive interpretation of a pronoun directed to the middle (see the Appendix for an example). In such cases, though, the midpoint of the two loci is in the middle of the signing space, which 


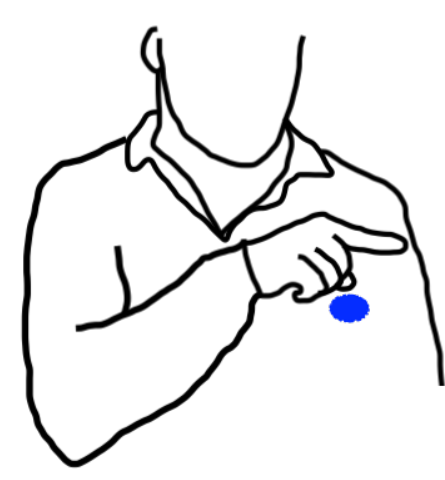

Figure 3 Directing the pronoun IX to the midpoint of two loci

opens the possibility that that this an instance of a 'default' or 'neutral' pronoun, which may act differently from pronouns signed at loci (cf. Schlenker 2011; Neidle, Kegl, MacLaughlin, Bahan \& Lee 2000: 94). Having the two relevant loci off-center allows one to establish that the pronoun is directed towards the midpoint of two localized antecedents.

NEXT-YEAR ELECTION PRESIDENT, WHO GOING-TO WIN? PERSON-a WHITE WIN []-nOt. PERSON-b BLACK OR PERSON-c ASIAN COMPETE WIN. THEN IX-midpoint:b,c ESTABLISH LAW EQUALITY CITIZEN.

'In next year's presidential election, who will win? A white person won't win; a Black person or an Asian person will win. They will then establish a law on citizen equality.'

Pronouns at the midpoint of two loci provide a second challenge to the analysis of Schlenker (2011). For Schlenker (2011), a discourse referent is established at each locus at which an antecedent DP is signed, but no mechanism is provided to introduce a discourse referent at the new locus between these points in space.

\subsection{Challenge \#3: Verb agreement}

In spoken language, constraints on morphosyntactic features appear not only for pronouns, as seen above in (15), but also for verb agreement. English has relatively few paradigms of verbal agreement, but the point can be illustrated with person agreement of the verb be. In (23), I is 1st person singular and you is 2nd person singular; as a result, it is unclear how to agree the verb with the disjunctive subject you or $I$, as there is no singular form of be that is unspecified for person. In contrast, the modal verb will is invariant; it is thus unspecified for person, so can appear perfectly naturally with the disjunctive subject. 


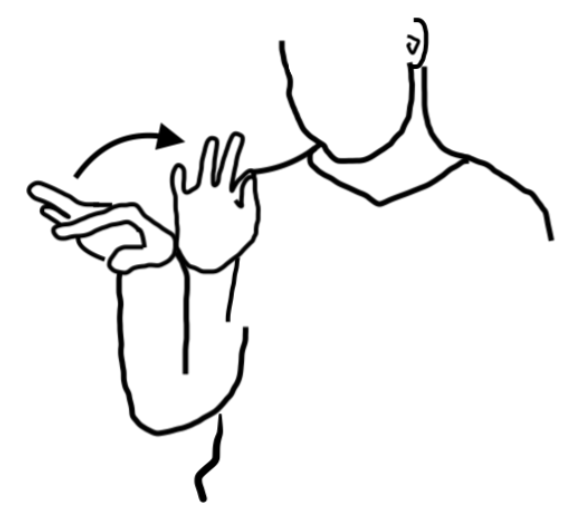

Figure 4 The verb CHOOSE, with a 1st person subject and 3rd person direct object at a locus on the right

(23) a. Either you or I $\left\{*\right.$ is $/ *$ am $/{ }^{\text {? }}$ are $\}$ wrong.

b. Either you or I will take care of it.

Johnson \& Bayer (1995) discuss a similar example involving case-marking in German. The verb findet, 'find', takes an object in accusative case and the verb hilft, 'help', takes an object in dative case. As a result, when the two verbs are coordinated, it is impossible to use an object with either accusative or dative case when the two forms differ, as in (24a). On the other hand, some nouns have a syncretism of the accusative and dative form; they are thus underspecified for case, so can be used grammatically with the coordinated verb, as in (24b).
a. * Er findet und hilft \{Männer / Männern\}. He finds and helps $\{$ men.ACC / men.DAT
b. Er findet und hilft Frauen. He finds and helps women.ACC/DAT 'He finds and helps women.'

(German; Johnson \& Bayer 1995)

Sign language, too, shows patterns of verbal agreement. In LSF, like in many other sign languages, many verbs are directional verbs, which move in space between the loci of their arguments. The verb GIVE, for example, moves in space from the locus of the subject (the giver) to the locus of the indirect object (the receiver). The verb CHOOSE moves in space from the locus of the direct object (the entity chosen) to the locus of the subject (the chooser), as shown in Figure 4. Other verbs, such as PREFER, are plain verbs, which are invariant in form.

At a first pass, the descriptive generalizations for verb agreement mirror exactly what was observed in Section 3.2 for pronouns. Specifically, when two disjuncts 
are indexed at different loci, (a) non-agreeing forms are possible, (b) agreement is possible with the midpoint of the two loci, but (c) agreement with either disjunct is not possible-with exceptions, to be discussed.

In examples (25-27), a disjoined DP has been topicalized. In example (25), the verb is the plain verb PREFER. As a consequence, even though the disjuncts are signed at two different loci, the sentence is grammatical and has a disjunctive meaning: the speaker will choose one of those two people. Examples with plain verbs like PREFER parallel examples with null pronouns in Section 3.2: in both cases, the forms are unspecified for locus, so can agree with a disjunctive DP. (Below, the sign glossed PI is a pronominal form; see Schlenker 2018 for discussion of its precise semantics.)

(25) MYSELF JUDGE. CL-3-come PERSON PERSON PERSON. PI-a NAME1 OR PI-b NAME2, IX-1 PREFER.

'I am the judge; three people came forward. I prefer Name1 or Name2.'

In example (26), the first disjunct is established to the left of the signer and the second disjunct is established in front of the signer. The directional verb CHOOSE agrees with the midpoint of the two loci, slightly left of center. Once again, the sentence is grammatical and has a disjunctive meaning: the speaker will choose one of those two people.

MYSELF JUDGE. THREE PERSON PERSON PERSON CL-3-come. PI-a NAME1 OR PI-b NAME2, IX-1 CHOOSE-midpoint:a,b.

'I am the judge; three people came forward. I will choose Name1 or Name2.'

On the other hand, if the verb agrees with either of the two loci, as in (27), the pattern of judgments changes. Grammaticality judgments of the sentence become degraded, and, to the extent it is grammatical, the meaning of the sentence changes. The topicalized disjunct is interpreted as a further restriction on the domain, not as an argument of the verb: of those two people, the speaker will choose that one.

?? MYSELF JUDGE. THREE CL-3-come PERSON-a PERSON-b PERSON-c. PI-a NAME1 OR PI-b NAME2, IX-1 CHOOSE-a.

'I am the judge; three people came forward. Of Name1 and Name2, I will choose Name1.'

In spoken language, when a coordination structure shows a morphological mismatch, one strategy of agreement that is sometimes observed is Closest Conjunct Agreement (Nevins \& Weisser 2018). In such patterns, the verb simply agrees with the coordinated DP that is structurally closest. In the Welsh sentence in (28), the subject of the sentence is the coordination ti a Megan, 'you and Megan'. The complex 
DP is a 2nd person plural, as seen by the agreement of the pronoun ein hunain, 'yourselves'. Nevertheless, the verb shows 2nd person singular marking, agreeing with the structurally closest of the two conjuncts, $t i$, 'you', which immediately follows the verb.

(28) Gwelais [ti a Megan $]$ ein hunain see.PAST.2SG you.SG and Megan 2PL self

'You and Megan saw yourselves.' （Welsh; via Nevins \& Weisser 2018)

Similar examples can arguably be created in English in constructions with expletive subjects or locative inversion, as in (29), where the verb lives agrees with the singular old man as opposed to the plural coordination structure.

(29) In the house at the top of the hill lives an old man and his wife.

An exactly parallel pattern can be observed in LSF. Specifically, when the disjoined object in (27) is not topicalized, the verb may still be unspecified for locus or agree with the midpoint of the the two loci, but it may also agree with the locus of the structurally closer disjunct. The sentence in (30a) is grammatical, with a disjunctive interpretation of the direct object. In contrast, agreement with the locus of the second disjunct makes the sentence ungrammatical, as seen in (30b). We note that the acceptability of (30a) cannot be explained simply as phonetic assimilation of an underspecified form, since the verb CHOOSE moves away from the locus of the direct object.

(30) a. MYSELF JUDGE; CL-people TEN. IX-1 1-CHOOSE-a PI-a NAME1 OR PI-b NAME2.

'I'm the judge; there are ten people. I'll choose Name1 or Name 2.'

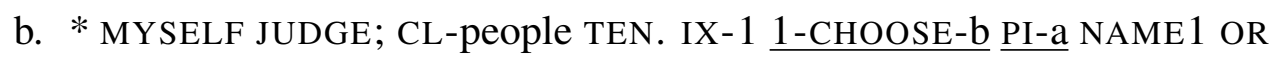
$\underline{\text { PI-b }}$ NAME2.

These patterns of verbal agreement pose a third challenge to Schlenker's semantic analysis of loci. On the one hand, a semantic analysis can in principle capture the basic pattern observed in (25-27), by analyzing agreement markers as presuppositions on the arguments of the verb. On the other hand, patterns of Closest Conjunct Agreement, in which the use of loci is governed by structural considerations, cannot be explained by a fully semantic analysis of loci.

\subsection{Summary of challenges}

In this section, we have presented three challenges to a theory on which loci are the overt spell-out of dynamic variables. First, in cases with split antecedents, we 
saw examples in which a single locus indexes multiple individuals. This is not possible if loci are variables, since assignment functions by definition map each variable to a unique individual. Second, in cases with disjuncts at different loci, we observed the need for a mechanism to introduce discourse referents at the midpoint of two disjuncts. No such mechanism is provided on the basic theory of loci as variables. Finally, in cases of verb agreement, we observed cases of Closest Conjunct Agreement, which cannot be analyzed by a fully semantic analysis.

In the next section, we propose an analysis to account for these three facts, in which locus interpretation is mediated via a featural layer that iconically preserves mereological properties.

\section{Proposal: a featural level with an iconic mereology}

To address the challenges introduced in Section 3, I propose a new system in which spatial loci are mediated by a featural layer, pronouns may denote sums of discourse referents, and the spatial features of these sum individuals are iconically determined.

\subsection{A featural layer}

Kuhn (2016), based on examples with quantificational binding, argues that sign language loci are not themselves variables; rather, they act like morphological phifeatures. Specifically, he shows that that multiple individuals can be indexed at the same locus, just as multiple individuals may all share the same gender feature.

Sentence (31) illustrates Kuhn's arguments (from ASL) with an analogous example in LSF. In English, when two pronouns appear under only, either one can in principle be bound or free, thus generating four possible readings, including two mixed readings in which one pronoun co-varies in the focus alternatives of only but the other does not. Kuhn's observation is that exactly the same readings are available in sign language, even when both pronouns are indexed at the same locus. The loci used in (31) are thus closer in behavior to gender features in (32) than to the covert indices used in the logical form in (33).

ONLY JEAN QUESTION POSS-a MOTHER POSS-a FAVORITE COLOR.

'Only Jean quizzed his mother about his favorite color.'

Only Jean quizzed his ${ }_{[\mathrm{masc}]}$ mother about his ${ }_{[\mathrm{masc}]}$ favorite color. [only $\mathrm{Jean}_{j}$ ] $\lambda x$.x [ask $j$ 's mother $x$ 's favorite color.]

Here, we extend this insight to dynamic binding as well: loci are represented in the grammar as morphosyntactic features, which constrain interpretation but do not force co-reference. For several ways to implement this insight, see Kuhn (2016), Steinbach \& Onea (2016), and Schlenker (2016). 


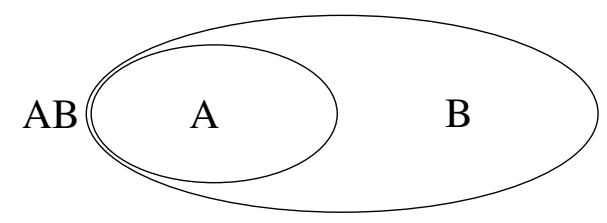

Figure 5 Establishing a plural DP at superset locus AB and subset locus A makes reference possible to the complement set at locus B.

\subsection{An iconic mereology of loci}

To this analysis, we add the assumption that the mereology of discourse referents is represented iconically - that is, mereological structure of the denotation is reflected in spatial structure of loci.

Such a proposal has been made previously in the literature based on plurals, which are signed by moving a pronoun over an area of space. Schlenker, Lamberton \& Santoro (2013) show that when one plural is indexed at a sub-area of a second plural, this generates the inference that the former is a subset of the latter, and simultaneously establishes the locus of three discourse referents: the superset, the subset, but also the complement set, as illustrated in Figure 5. The locus of this third discourse referent is thus determined iconically by the placement of the first two.

Here, I propose that this iconic mereology, easily observable for plurals, also extends to singular reference. Of particular note, we have seen that disjunctive reference may be analyzed as using sums of discourse referents, even when these sums may denote an atomic individual. I propose that the same fundamental principles govern the placement of the locus of $x+y$ regardless of whether $x+y$ denotes a plurality ('the boys ${ }^{x}$ and the girls ${ }^{y}$ ') or an atomic individual ('a boy ${ }^{x}$ or a girl' ${ }^{y}$ ').

Concretely, I propose that all loci are areas of space; singular pronouns point to the middle of an area (cf. Steinbach \& Onea 2016). We add the following iconic principle: if $x$ at is indexed at locus a and $y$ at locus $\mathrm{b}$, then $x+y$ is indexed at $\mathrm{a}+\mathrm{b}$. As a special case, if $x$ and $y$ are both at locus a, then $x+y$ is indexed at a $(=\mathrm{a}+\mathrm{a})$. Finally, we provide the conditions of use of loci by analogy with those of gender features. The feminine pronoun she must be anaphoric to an index that bears the feature [fem]; it presupposes that the value of this index is non-empty in the local context. Likewise, IX-a must be anaphoric to an index that bears the feature [a]; it presupposes that the value of this index is non-empty in the local context.

\subsection{Addressing the challenges}

This analysis accounts for the challenges presented in Section 3. First, regarding patterns of verbal agreement, since loci are mediated by a featural layer, they interact 
in the grammatical system like any other features (e.g., as uninterpreted features that must be checked or as presuppositions on arguments). As a consequence, any morphosyntactic theory of Closest Conjunct Agreement in spoken language will transfer directly over to the case of loci.

Second, regarding midpoint pointing, we have argued that pronouns can refer to any (non-empty) sum of discourse referents. According to our iconic mereology of space, pointing 'between' two loci is in fact pointing at the sum of the referents at the two loci. Just like in the case of complement set anaphora, the locus of the new discourse referent is determined iconically by the placement of the first two. In the case of disjunctive antecedents, the denotation of this sum is whichever disjunct has the relevant property.

Finally, regarding disjoined sentences, we observe that the proposal makes no reference to the syntactic constituent ' $\mathrm{X}$ or $\mathrm{Y}$ '; disjunctive discourse referents may have a split antecedent. The featural layer allows these two discourse referents to be indexed at the same locus. In this case, their sum is also at this locus.

\subsection{Comparison to spoken language}

One of the theory-neutral generalizations that emerges from the present paper is that there are strong parallels between the behavior of loci in sign language and the behavior of related phenomena in spoken language. The fact that a midpoint locus can be used when disjuncts are at different loci is parallel to the fact that English singular they can be used with unspecified gender reference (e.g. a boy or a girl ... they ...). Cases in which two individuals share the same locus are parallel to examples where agreement is only possible when two coordinated forms participate in a syncretism (e.g. findet und hilft Frauen).

In spoken language, one proposal that has been made to account for featural underspecification is the idea that feature checking doesn't require matching; it requires subsumption (Bernardi \& Szabolcsi 2008). Thus, English singular they, underspecified for gender, subsumes the features of both he and she. From this perspective, we could say that LSF IX-midpoint:a,b subsumes the features of IX-a and IX-b. The difference for sign language is that feature subsumption is represented iconically in space.

\section{Conclusion}

In this paper, we explored the representation of discourse reference and its relation to spatial loci in sign language via a number of novel examples involving disjunction. An initial analysis by Schlenker (2011) was shown to face several challenges involving locus reuse, an iconically innovative use of space, and verbal agreement 
paradigms. We argued that extending insights from Kuhn (2016) to dynamic cases resolves these challenges for Schlenker (2011) while preserving its essential arguments. The proposal consisted of the following primary elements: (a) loci are mediated by a featural layer; (b) in spoken and sign language, pronouns may denote sums of discourse referents; (c) the spatial features of these sum individuals are iconically determined.

\section{Appendix: Numerical ratings of LSF sentences}

Table 1 presents numerical ratings for the LSF sentences presented in this paper. Additionally, sentences (17), (18), (19), (20), and (22) were replicated with a second paradigm of the same structure but with new lexical items and a possessive pronoun, shown below; ratings of these sentences are provided as well. The pronoun glossed POSS-flick has a slightly different movement than IX-alternating (a flick between the two loci), but generates the same pattern of syntactic and semantic judgments.

$(17)^{\prime}$ CONTEST SCHOOL BEST ONE PLACE WHO WIN? [STUDENT SCIENCE]-a OR [STUDENT MATH]-b. THEN POSS-a BEST FILE COMPETE OTHER SCHOOL. 'Who will win the school competition? A science student or a math student. The science student's file will then compete against other schools.'

$(18)^{\prime}$ NOW SCHOOL CONTEST STUDENT ADVANCE REMAIN TWO PERSON PERSON, JEAN-a OR PIERRE-b. WELL, DIRECTOR GOING-TO CHOOSE. THEN $\underline{\emptyset}$ FILE COMPETE OTHER SCHOOL EACH. LATER TWO-MONTH FIRST PLACE. 'Two people are now left in the school competition: Jean and Pierre. The director will choose. The winner's file will then compete against other schools. In two months, the first place winner is chosen.

(19)' CONTEST SCHOOL BEST PLACE WHO WIN? [STUDENT MATH]-a OR [STUDENT SCIENCE]-b. THEN POSS-flick-a,b FILE COMPETE OTHER SCHOOL.

'Who will win the school competition? A math student or a science student. The winner's file will then compete against other schools.'

(20)' CONTEST SCHOOL BEST ONE PLACE WHO WIN? [STUDENT MATH]-a OR [STUDENT SCIENCE]-b. THEN THE-TWO-a,b FILE BEST COMPETE OTHER SCHOOL.

'Who will win the school competition? A math student or a science student. The two students' files will then compete against other schools.'

$(22)^{\prime}$ CONTEST SCHOOL BEST PLACE WHO WIN? [STUDENT SCIENCE]-a OR [STUDENT MATH]-b. THEN POSS-midpoint:a,b FILE BEST COMPETE OTHER SCHOOL.

'Who will win the school competition? A science student or a math student. The winner's file will then compete against other schools.' 


\begin{tabular}{c|l|c||c|c|c|c}
\hline Example & Filename & Average & Rating 1 & Rating 2 & Rating 3 & Rating 4 \\
\hline$(14)$ & $71>15$ & 7 & 7 & 7 & 7 & \\
$(17)$ & $47>9$ & 6 & 5 & 6 & 7 & \\
$(17)^{\prime}$ & $50>6$ & 4.8 & 5 & 5 & 4 & 5 \\
$(18)$ & $72>25$ & 7 & 7 & 7 & & \\
$(18)^{\prime}$ & $42>0333$ & 6.7 & 7 & 6 & 7 & \\
$(19)$ & $47>11$ & 7 & 7 & 7 & 7 & \\
$(19)^{\prime}$ & $50>4$ & 7 & 7 & 7 & 7 & 7 \\
$(20)$ & $72>1$ & 7 & 7 & 7 & & \\
$(20)^{\prime}$ & $50>18$ & 7 & 7 & 7 & 7 & 7 \\
$(22)$ & $72>27$ & 7 & 7 & 7 & & \\
$(22)^{\prime}$ & $50>5$ & 6.3 & 6 & 7 & 5 & 7 \\
$(25)$ & $36>0063$ & 7 & 7 & 7 & 7 & \\
$(26)$ & $36>0069$ & 6.7 & 7 & 7 & 6 & \\
$(27)$ & $36>0064$ & 3.3 & 1 & 5 & 4 & \\
$(30 a)$ & $36>0047$ & 6.7 & 7 & 7 & 6 & \\
$(30 b)$ & $36>0045$ & 1 & 1 & 1 & 1 & \\
$(31)$ & $05>3390$ & 7 & 7 & 7 & &
\end{tabular}

Table 1 Numerical ratings of elicited LSF sentences on a 7-point scale (7=best)

\section{References}

Bernardi, Raffaella \& Anna Szabolcsi. 2008. Optionality, scope, and licensing: an application of partially ordered categories. Journal of Logic, Language, and Information 17(3). 237-283. doi:10.1007/s10849-008-9060-y.

Dekker, Paul. 1993. Transsentential meditations: ups and downs in dynamic semantics: University of Amsterdam PhD dissertation.

Elbourne, Paul. 2005. Situations and Individuals Current Studies in Linguistics. Boston, MA: MIT Press.

Evans, Gareth. 1980. Pronouns. Linguistic Inquiry 11(2). 337-362. http://www. jstor.org/stable/4178164.

Groenendijk, Jeroen \& Martin Stokhof. 1991. Dynamic predicate logic. Linguistics and Philosophy 14(1). 39-100. doi:10.1007/BF00628304.

Heim, Irene. 1982. The Semantics of Definite and Indefinite Noun Phrases. Amherst, MA: University of Massachusetts PhD dissertation.

Heim, Irene. 1990. E-type pronouns and donkey anaphora. Linguistics and Philosophy 13(2). 137-178. doi:10.1007/bf00630732.

Johnson, Mark \& Sam Bayer. 1995. Features and agreement in lambek categorial grammar. Formal Grammar Workshop 123-137. 
Disjunctive discourse referents in LSF

Kamp, Hans. 1981. A theory of truth and semantic representation. In Jeroen Groenendijk, Theo Janssen \& Martin Stokhof (eds.), Formal Methods in the Study of Language, vol. 135 Mathematical Center Tracts, 277-322. Amsterdam, Netherlands.

Kuhn, Jeremy. 2016. ASL Loci: Variables or Features? Journal of Semantics 33(3). 449-491. doi:10.1093/jos/ffv005.

Lillo-Martin, Diane \& Edward Klima. 1990. Pointing out differences: ASL pronouns in syntactic theory. In Susan Fischer \& Patricia Siple (eds.), Theoretical Issues in Sign Language Research, vol. 1, 191-210. Chicago, IL: University of Chicago Press.

Neidle, Carol, Judy Kegl, Dawn MacLaughlin, Benjamin Bahan \& Robert G. Lee. 2000. The Syntax of American Sign Language: Functional Categories and Hierarchical Structure. Cambridge, MA: MIT Press.

Nevins, Andrew \& Philipp Weisser. 2018. Closest conjunct agreement. Annual Review of Linguistics 5. 219-241. doi:10.1146/annurev-linguistics-011718012708.

Schlenker, Philippe. 2011. Donkey anaphora: the view from sign language (ASL and LSF). Linguistics and Philosophy 34(4). 341-395. doi:10.1007/s10988011-9098-1.

Schlenker, Philippe. 2016. Featural variables. Natural Language and Linguistic Theory 34. 1067-1088. doi:10.1007/s11049-015-9323-7.

Schlenker, Philippe. 2018. Strong pronominals in ASL and LSF? Sign Language \& Linguistics 21(2). 380-390. doi:10.1075/s1l.00025.sch.

Schlenker, Philippe, Jon Lamberton \& Mirko Santoro. 2013. Iconic variables. Linguistics and Philosophy 36(2). 91-149. doi:10.1007/s10988-013-9129-1.

Steinbach, Markus \& Edgar Onea. 2016. A DRT analysis of discourse referents and anaphora resolution in sign language. Journal of Semantics 33(3). 409-448. doi:10.1093/jos/ffv002.

Stone, Matthew D. 1992. Or and anaphora. In Chris Barker \& David Dowty (eds.), 2nd Semantics and Linguistic Theory Conference (SALT 2), 367-385. Columbus, OH: Ohio State Department of Linguistics. doi:10.3765/salt.v2i0.3037.

Jeremy Kuhn

Institut Jean Nicod

Ecole Normale Supérieure

29, rue d'Ulm

75005 Paris, France

jeremy.d.kuhn@gmail.com 